\title{
DISEÑO Y DESARROLLO DE QUESO FRESCO ENRIQUECIDO CON ACEITE DE SOYA
}

\author{
DESIGN AND DEVELOPMENT OF FRESH CHEESE ENRICHED WITH SOJA OIL
}

\author{
${ }^{1}$ Tuesta Ch., Tarsila; ${ }^{2}$ Vargas M., Jorge; ${ }^{3}$ García G., Gilberto; ${ }^{4}$ Ureña P., Milber y ${ }^{5}$ Neira M. Enrique
}

\begin{abstract}
Resumen
El objetivo fue obtener un queso fresco enriquecido con aceite de soya, con aceptabilidad sensorial y rico en grasas poliinsaturadas, principalmente los ácidos grasos esenciales linoleico y linolénico. Como materias primas se utilizó: leche fresca descremada de vacas de raza Holstein y aceite de soya. El contenido de ácidos grasos poliinsaturados, que contiene el queso fresco elaborado con leche entera se elevó de 5,01\% a 54,83\% al elaborarlo con leche descremada enriquecida con $2 \%$ de aceite de soya. Se evalúo las propiedades sensoriales del queso fresco enriquecido correspondiendo una formulación óptima con: sal $14 \mathrm{~g} / \mathrm{L}$ y aceite $2 \%$; obteniéndose valores de: Sabor 8,870, Textura 8,237 y Apariencia 9,013 sobre un máximo de 10,0 unidades. La vida útil del queso fresco enriquecido con aceite de soya fue de 23 días. Se concluye que es factible la producción del queso fresco enriquecido con ácidos grasos poliinsaturados y se recomienda su fabricación.
\end{abstract}

Palabras Clave: Queso Fresco, Aceite de Soya, Ácidos Grasos Esenciales, Evaluación Sensorial, Vida Útil.

\begin{abstract}
The objective was to obtain a fresh cheese enriched with soja oil, with sensorial acceptability and rich in polyunsaturated fats, mainly the essential fatty acids linoleic and linolenic. Skimmed fresh milk from Holstein cows and soja oil were used as raw materials. The content of polyunsaturated fatty acids inside of the fresh cheese elaborated with whole milk increased from 5,01\% to 54,83\% when elaborating it with skimmed milk enriched with $2 \%$ of soja oil. Sensorial properties of enriched fresh cheese were evaluated corresponding the best formulation with: salt $14 \mathrm{~g} / \mathrm{L}$ and oil $2 \%$; obtaining values of: Flavor 8,870, Texture 8,237 and Appearance 9,013 over a maximum of 10,0 units. The shelf life of the fresh cheese enriched with soja oil was 23 days. We conclude that it is feasible the production of fresh cheese enriched with polyunsaturated fatty acids and its production is recommended.
\end{abstract}

Key words: Fresh cheese, Soja Oil, Essential Fatty Acids, Sensorial Evaluation

\section{Introducción}

Las dietas con alto contenido de ácidos grasos saturados y bajo en ácidos grasos poliinsaturados, favorecen el origen de problemas cardiovasculares (Leighton, 2002). En nuestro país la tasa estimada de mortalidad a causa de enfermedades del sistema circulatorio es de 114,5 por cada 100000 habitantes (INEI, 1999 citado por MINSA, 2002), Asimismo la cantidad de alimentos consumidos como fuentes ricas en ácidos grasos poliinsaturados tales como; huevos de codorniz, leches enriquecidas, yogurt, etc., es muy baja, lo cual responde a cuestiones de hábito alimentario, formas de su preparación, así como también la baja disponibilidad de estos productos y algunos no están al alcance económico de las clases necesitadas. La mejor forma de aumentar el aporte de ácidos grasos poliinsaturados en la dieta del ser humano, es consumiendo pescados y mariscos. También pueden consumirse alimentos enriquecidos con aceites de pescado, pero su consumo está limitado ya que se asocia a olores desagradables. Otra alternativa de alimentación con fuentes de ácidos grasos poliinsaturados se da a través de aceites y semillas ricas en ácidos alfa linolénico y linoleico denominados ácidos grasos esenciales, tales como el aceite de soya, canola y de linaza; en el caso de estos dos últimos su importación es limitado por sus altos precios (MINAG, 2007). En nuestro país, el queso fresco ocupa el segundo lugar en producción y consumo de quesos (MINAG, 2007) y su elaboración se lleva a cabo teniendo como materia prima leche fresca proveniente de diversas razas de ganado vacuno. Su consumo es de $2 \mathrm{~kg}$ per cápita por año (INEI, 1997; citado por Padilla, 1999) cifra creciente pero que es muy inferior al consumo de países como Francia; con $23 \mathrm{~kg}$ per cápita anual. La elaboración de un producto enriquecido en ácidos grasos poliinsaturados teniendo como materia prima leche fresca, se sustenta por la existencia de un importante volumen de producción sin proceso industrial (40\%). La

\footnotetext{
${ }^{1}$ Universidad Nacional de Ingeniería, Lima, Perú. E-mail: tarsilat@hotmail.com

${ }^{2}$ Universidad Nacional Agraria La Molina, Lima, Perú. E-mail: jvargasm@lamolina.edu.pe

${ }^{3}$ Universidad Nacional de Ingeniería, Lima, Perú.

${ }^{4}$ Docente Asociado, D.I.A.P.A. Univ. Nac. Agraria La Molina

${ }^{5}$ Universidad Nacional de Ingeniería, Lima, Perú.
} 
elaboración de queso fresco a partir de leche desgrasada, proveniente de vacas Holstein, es un producto alternativo posible de enriquecerlo con ácidos poliinsaturados por su fácil consumo por parte de la población.

El objetivo del presente trabajo fue obtener un queso fresco enriquecido con aceite de soya, con aceptabilidad sensorial y rico en grasas poliinsaturadas, principalmente los ácidos grasos esenciales linoleico y linolénico.

\section{Materiales y métodos}

La elaboración y análisis físico químico de la leche y quesos frescos se hizo en el Laboratorio de Leche del Departamento de Producción Animal, Facultad de Zootecnia-UNALM. Los análisis del producto terminado óptimo, se efectuó en el Laboratorio de INASSA: Análisis de Grasas; en el Laboratorio Marino Tabusso UNALM se realizó el Análisis Microbiológico. Las pruebas de Análisis Sensorial se efectuaron con el personal perteneciente a las siguientes instituciones; Facultad de Zootecnia-UNALM, Facultad de Ingeniería Química y Textil-UNI e ISTP "Escuela Superior Privada de Tecnología SENATI". Se utilizó leche proveniente de vacas Holstein del establo de la UNALM, aceite de soya comercial, sal yodada, cloruro de calcio: grado USP, coagulante: CHY-MAX, CHR HANSEN.

Para cada muestra de leche se determinó: Grasa (ITINTEC, 1982), densidad y acidez (ITINTEC, 1982), análisis que se llevaron a cabo durante la elaboración del queso tanto en la etapa preliminar como en la etapa experimental, realizándose los siguientes análisis: Grasa: Queso. Determinación de Materia Grasa, se realizó según NTP 202.072, INDECOPI (1982). Composición de Ácidos Grasos: Mediante ISO (1978) e ISO (1990). Análisis Microbiológico: Se utilizaron $1000 \mathrm{~g}$ por muestra para análisis cada 5 días durante un período de 23 días, realizándose las determinaciones de recuento de mesófilos aerobios o facultativos viables (UFC/g). A los 23 días de almacenamiento se determinó el recuento de Coliformes, Sthaphilococcus y Salmonellas (NMP/g). En cumplimiento de la Norma Técnica Peruana (ITINTEC, 1982) se los requisitos microbiológicos de un queso fresco. Análisis Sensorial de las Formulaciones: Se usó $30 \mathrm{~g}$ de queso por juez. Cada formulación fue degustada por 100 personas, según la ASTM, (1968) citado por Ureña et al. (1999).

\section{Resultados y discusión}

Ensayos Experimentales Preliminares: El objetivo de la etapa preliminar fue determinar la capacidad de adsorción del aceite de soya en el queso. En la Tabla 1 se muestra la composición de ácidos grasos del aceite de soya.
Tabla 1. Composición de Ácidos Grasos del Aceite de Soya.

\begin{tabular}{lcr}
\hline $\begin{array}{r}\text { Composición de } \\
\text { Ácidos Grasos }\end{array}$ & $\begin{array}{c}\text { Unidad } \\
\text { Cn:m }\end{array}$ & $\begin{array}{r}\text { Resultados } \\
(\%)\end{array}$ \\
\hline Ácido Palmítico & $16: 0$ & 10,63 \\
Ácido Esteárico & $18: 0$ & 3,98 \\
Ácido Oleico & $18: 1$ & 20,94 \\
Ácido Linoléico & $18: 2$ & 56,22 \\
Ácido Araquídico & $20: 0$ & 0,46 \\
Ácido Eicosenóico & $20: 1$ & 0,33 \\
Ácido Linolénico & $18: 3$ & 6,51 \\
Ácido Erúcico & $22: 1$ & 0,62 \\
Ácido Lignocérico & $24: 0$ & 0,08 \\
Ácido Nervónico & $24: 1$ & 0,24 \\
Saturado & & 15,15 \\
Monoinsaturado & & 22,13 \\
Poliinsaturado & & 62,73 \\
\hline \multicolumn{1}{c}{ Total } & & 100,01
\end{tabular}

$\mathrm{n}=$ número de carbonos

$\mathrm{m}=$ número de dobles enlaces

w=Omega

Método

Composición de Ácidos Grasos: ISO 5509-1978

La proporción de ácidos grasos insaturados del aceite de soya utilizado se muestran en la Fig. 1.

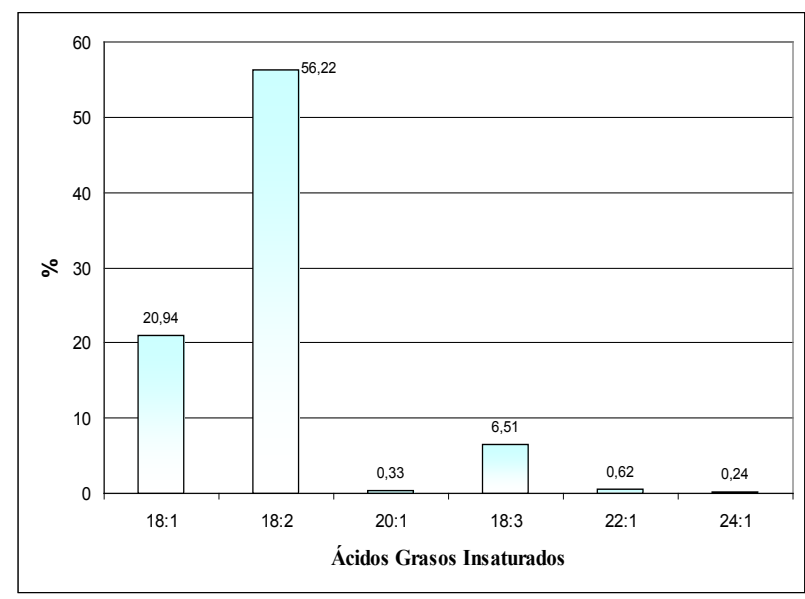

Figura 1. Composición de Ácidos Grasos Insaturados en Aceite de Soya.

La presencia de los ácidos linoleico y linolénico que sumados representan el 62,73\% de los ácidos grasos del aceite de soya lo hace valioso para ser utilizado en las experiencias de enriquecimiento del queso fresco.

Elaboración de los Quesos Muestras: Estos fueron elaborados siguiendo el proceso convencional. La particularidad en su elaboración consistió en conseguir que 
el aceite se incorpore en el coágulo del queso, formando una malla que reduzca el arrastre de aceite por parte del suero. Mediante tres ensayos aleatorios, y teniendo como parámetro indicador el mayor o menor proporción de arrastre de aceite, se determinó que el aceite debería incorporarse entre las etapas de pasteurizado y mezcla mecánica, verificado por los ensayos experimentales preliminares de los lotes que se indican en la Tabla 2.

Tabla 2. Lotes de queso fresco enriquecido elaborados.

\begin{tabular}{ccccc}
\hline \multirow{2}{*}{ Lote } & Queso & Leche & \multicolumn{2}{c}{ Aceite } \\
& $\mathrm{kg}$ & $\mathrm{mL}$ & $\mathrm{mL}$ & $\%$ \\
\hline Control & 0,5 & 4000 & 0 & 0,0 \\
1 & 3,5 & 28000 & 28 & 0,1 \\
2 & 3,5 & 28000 & 280 & 1,0 \\
\hline
\end{tabular}

\section{Medición del Contenido de Grasa en los Quesos Frescos Muestras Elaborados}

El queso muestra fue seccionado en cuatro parte es como se muestra en la Fig 2.

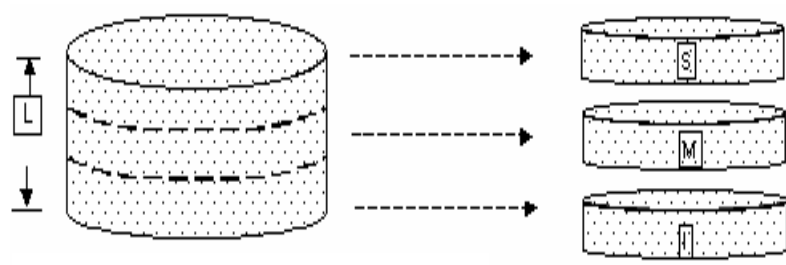

Figura 2 Molde de Queso seccionado para determinación de grasa.

Los análisis se realizaron durante seis días consecutivos. Los resultados obtenidos se muestran en las Tablas 3, 4 y 5 .

Tabla 3. Contenido de Grasa en Queso Fresco, sin Aceite Adicionado, de acuerdo a sección de muestreo.

\begin{tabular}{ccccc}
\hline Número de Días & $* \mathrm{~S}$ & $* \mathrm{M}$ & $* \mathrm{I}$ & ${ }^{* \mathrm{~L}}$ \\
\hline Día $1^{\text {ro }}(24 \mathrm{H})$ & 12,5 & 13 & 12 & 11,5 \\
\hline
\end{tabular}

Tabla 4. Contenido de Grasa en Queso Fresco Enriquecido con $0,1 \%$ de Aceite Adicionado, de acuerdo a sección de muestreo

\begin{tabular}{ccccc}
\hline Días & $* \mathrm{~S}$ & $* \mathrm{M}$ & $* \mathrm{I}$ & $* \mathrm{~L}$ \\
\hline Día ${ }^{\mathrm{ro}}$ & 12 & 12,5 & 12,0 & 11,5 \\
Día 2 & 13 & 11,5 & 12,5 & 10,5 \\
Día $^{\text {ro }}$ & 13 & 12,0 & 12,5 & 13,0 \\
Día $^{\text {to }}$ & 13 & 11,5 & 13,0 & 13,0 \\
Día $^{\text {to }}$ & 13 & 12,0 & 14,5 & 13,0 \\
Día $^{\text {to }}$ & 13 & 12,5 & 14,0 & 14,0 \\
\hline
\end{tabular}

Tabla 5 Contenido de Grasa en Queso Fresco Enriquecido con $1,0 \%$ de Aceite Adicionado, de acuerdo a sección de muestreo.

\begin{tabular}{|c|c|c|c|c|}
\hline Días & $* \mathrm{~S}$ & $* \mathrm{M}$ & $* \mathrm{I}$ & $* \mathrm{~L}$ \\
\hline Día $1^{\text {ro }}$ & 15,5 & 12,0 & 15,0 & 14,0 \\
\hline Día $2^{\text {do }}$ & 15,5 & 14,0 & 17,0 & 15,5 \\
\hline Día $3^{\text {ro }}$ & 14,5 & 13,5 & 16,5 & 15,0 \\
\hline Día $4^{\text {to }}$ & 15,0 & 16,0 & 17,0 & 16,0 \\
\hline Día $5^{\text {to }}$ & 14,0 & 14,0 & 17,0 & 14,5 \\
\hline Día $6^{\text {to }}$ & 15,0 & 13,0 & 17,0 & 16,5 \\
\hline
\end{tabular}

\section{ENSAYOS EXPERIMENTALES FINALES DISEÑO EXPERIMENTAL}

Comprobado que el queso fresco si tiene capacidad de adsorción de aceite de soya y definido el esquema de producción para la elaboración del queso fresco enriquecido, mediante ensayos experimentales finales se procedió a evaluar: Contenido de aceite de soya que se debe incorporar, Contenido de sal que se debe incorporar, la aceptabilidad sensorial del producto final y validar los trabajos experimentales mediante el análisis estadístico.

Las variables independientes fueron contenido de sal y de aceite que se añaden en cada formulación, así se tomaron contenidos de aceite entre 2 a $4 \%$ y contenido de sal entre 14 a $18 \mathrm{~g} / \mathrm{L}$. Haciendo uso del Diseño Factorial, se elaboraron las Tablas 6 y 7, donde la Tabla 7 muestra la formulación utilizada.

Tabla 6 Niveles de Concentración de sal y aceite.

\begin{tabular}{lrc}
\hline Nivel & Sal (g/L) & Aceite (\%) \\
\hline Bajo & 14 & 2 \\
Medio & 16 & 3 \\
Alto & 18 & 4 \\
\hline
\end{tabular}

Tabla 7 Planteamiento de las Formulaciones utilizadas.

\begin{tabular}{lcc}
\hline Formulaciones & $\begin{array}{c}\text { Concentración } \\
\text { de Sal } \\
(\mathrm{g} / \mathrm{L})\end{array}$ & $\begin{array}{c}\text { Porcentaje de Grasa } \\
(\%)\end{array}$ \\
\hline Formulación 1 & 18,0 & 2,0 \\
Formulación 2 & 18,0 & 4,0 \\
Formulación 3 & 16,0 & 3,0 \\
Formulación 4 & 14,0 & 4,0 \\
Formulación 5 & 18,0 & 3,0 \\
Formulación 6 & 16,0 & 4,0 \\
Formulación 7 & 16,0 & 2,0 \\
Formulación 8 & 14,0 & 3,0 \\
Formulación 9 & 14,0 & 2,0 \\
\hline
\end{tabular}

\section{Caracterización de la Leche Fresca de Vaca}

Considerando la sensibilidad de la leche a los factores ambientales, la caracterización de la leche fresca tanto entera como descremada se realizó en el momento mismo de preparar cada formulación. Los análisis se muestran en la Tabla 8 
Tabla 8 Análisis Físico Químico de Densidad y \% de Grasa en Leche Entera y Descremada .

\begin{tabular}{ccccc}
\hline \multirow{2}{*}{ Formulación } & \multicolumn{2}{c}{ Leche entera } & \multicolumn{2}{c}{ Leche descremada } \\
& $\mathbf{1}$ & $\mathbf{2}$ & $\mathbf{3}$ & $\mathbf{4}$ \\
& $\mathbf{d}(\mathbf{g} / \mathbf{m L})$ & Grasa $(\%)$ & $\mathbf{d}(\mathbf{g} / \mathbf{m L})$ & Grasa $(\%)$ \\
\hline F1 & 1,029 & 3,45 & 1,0333 & 0,2 \\
F2 & 1,0285 & 3,4 & 1,0319 & 0,3 \\
F3 & 1,0285 & 3,35 & 1,0315 & 0,5 \\
F4 & 1,0285 & 3,35 & 1,0315 & 0,5 \\
F5 & 1,0284 & 3,6 & 1,0331 & 0,2 \\
F6 & 1,0284 & 3,6 & 1,0329 & 0,2 \\
F7 & 1,0296 & 3,4 & 1,0319 & 0,4 \\
F8 & 1,0296 & 3,4 & 1,0319 & 0,4 \\
F9 & 1,0284 & 3,4 & 1,0316 & 0,1 \\
\hline
\end{tabular}

El análisis físico y químico de los quesos enriquecidos elaborados en base a las Formulaciones del F1 al F9, se muestran en las columnas 4, 5 y 6 de la Tabla 9.

Tabla 9. Análisis Físico Químico del Queso Fresco .

\begin{tabular}{|c|c|c|c|c|c|}
\hline \multirow{3}{*}{$\begin{array}{c}1 \\
\text { Formulación }\end{array}$} & \multirow{2}{*}{2} & \multirow{2}{*}{3} & \multicolumn{2}{|l|}{ Suero } & \multirow{2}{*}{$\begin{array}{c}\text { Queso } \\
6\end{array}$} \\
\hline & & & 4 & 5 & \\
\hline & Sal (g/L) & Grasa (\%) & Densidad $(\mathrm{g} / \mathrm{mL})$ & Grasa (\%) & Grasa (\%) \\
\hline$\overline{F 1}$ & 18 & 2 & 1,0232 & 0,40 & 14,0 \\
\hline F2 & 18 & 4 & 1,0238 & 0,30 & 17,5 \\
\hline F3 & 16 & 3 & 1,0232 & 0,50 & 20,0 \\
\hline $\mathrm{F} 4$ & 14 & 4 & 1,0230 & 0,60 & 24,0 \\
\hline F5 & 18 & 3 & 1,0230 & 0,30 & 17,0 \\
\hline F6 & 16 & 4 & 1,0232 & 1,00 & 22,5 \\
\hline F7 & 16 & 2 & 1,0214 & 0,30 & 9,5 \\
\hline F8 & 14 & 3 & 1,0248 & 0,45 & 12,5 \\
\hline F9 & 14 & 2 & 1,0248 & 0,20 & 10,5 \\
\hline
\end{tabular}

Enriquecido Se tomó como referencia que el queso fresco normal contiene de $0,5 \%$ hasta $1,0 \%$ de grasa en su suero, los resultados obtenidos presentados en la columna 5, muestran que estos se encuentran dentro de este rango. Lo cual demuestra que en todas las formulaciones experimentadas se ha realizado la adsorción del aceite incorporado.

La columna 6 de la Tabla 9 nos indica que el nivel de grasa que contienen los quesos enriquecidos "F7" y "F9" es menor que el del queso de leche entera sin aceite añadida, según la Tabla 3.

\section{Análisis Sensorial}

Contando con una población de 100 jueces no experimentados y mediante una escala hedónica se procedió la medición de las apreciaciones sensoriales: gusto de sal, textura y apariencia general, para cada una de las nueve formulaciones.

Los valores promedio de los atributos se presentan en la Tabla 10 y Fig 3 .
Tabla 10 Valores Promedios de los Atributos.

\begin{tabular}{cccc}
\hline Formulación & Gusto & Textura & Apariencia \\
\hline F1 & 7,4 & 6,7 & 8,4 \\
F2 & 7,9 & 7,3 & 8,2 \\
F3 & 7,4 & 7,1 & 7,5 \\
F4 & 7,2 & 6,5 & 7,3 \\
F5 & 6,8 & 6,3 & 7,9 \\
F6 & 6,5 & 5,4 & 7,5 \\
F7 & 7,0 & 7,7 & 8,5 \\
F8 & 6,5 & 6,6 & 7,1 \\
F9 & 8,9 & 8,2 & 9,0 \\
\hline
\end{tabular}

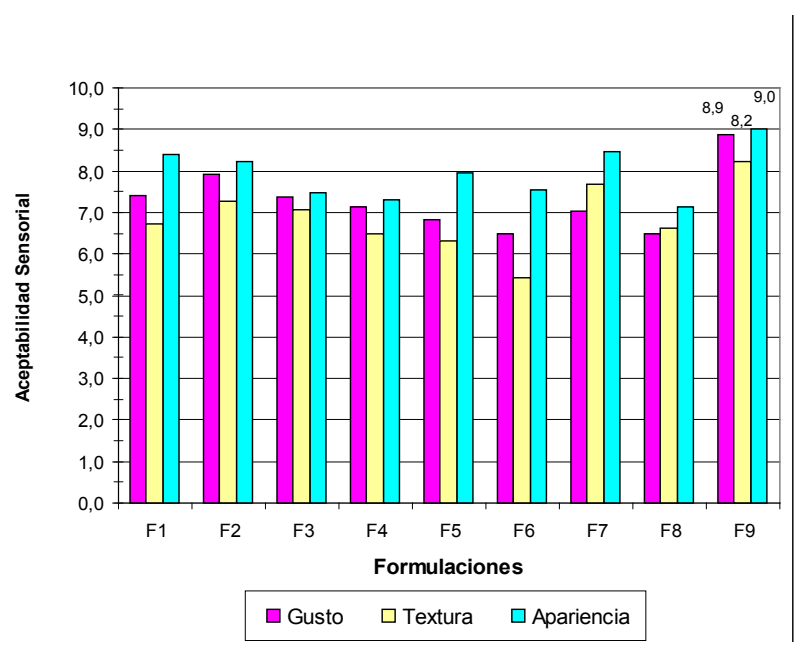

Figura 3. Aceptabilidad de las Formulaciones.

Podemos apreciar que la Formulación F9, presenta la mayor aceptabilidad en cuanto a los atributos evaluados. Por lo que fue necesario confirmar dichos resultados mediante un análisis estadístico.

\section{Análisis Estadístico}

La validación del Diseño Experimental desarrollado se efectúo mediante análisis estadístico haciendo uso del paquete STATGRAPHICS Plus 4, lo que permitió evaluar estadísticamente los atributos: Gusto de sal, Textura y Apariencia General, así como también determinar el óptimo de estos tres parámetros.

Para ello se elaboró un diseño factorial de tres niveles: diseño $3 \times 3$, que analizará los efectos de 2 factores en las 9 formulaciones. Los factores a considerar en sus valores máximos y mínimos son los que muestra la Tabla 7.

\section{Atributo Gusto de Sal}

A título ilustrativo se muestra solamente el análisis estadístico para el atributo gusto de sal para las nueve formulaciones. La Tabla 11 muestra los valores promedios de las 100 apreciaciones para cada una de las 9 formulaciones del atributo gusto de sal que contiene 
el queso. Se observa que la mejor formulación para este atributo es la número nueve.

Tabla 11. Formulaciones para Evaluar el Comportamiento de los Jueces en el Atributo Gusto de sal.

\begin{tabular}{cccc}
\hline Formulaciones & $\begin{array}{c}\text { Sal } \\
(\mathrm{g} / \mathrm{L})\end{array}$ & $\begin{array}{c}\text { Aceite } \\
(\%)\end{array}$ & Gusto de sal \\
\hline F1 & 18,0 & 2,0 & 7,42 \\
F2 & 18,0 & 4,0 & 7,91 \\
F3 & 16,0 & 3,0 & 7,37 \\
F4 & 14,0 & 4,0 & 7,15 \\
F5 & 18,0 & 3,0 & 6,83 \\
F6 & 16,0 & 4,0 & 6,48 \\
F7 & 16,0 & 2,0 & 7,03 \\
F8 & 14,0 & 3,0 & 6,49 \\
F9 & 14,0 & 2,0 & 8,87 \\
\hline
\end{tabular}

Tabla 12. Efectos Estimados para el Atributo Gusto de Sal.

\begin{tabular}{cc}
\hline & Efectos \\
\hline Promedio & $6,581+/-0,542$ \\
A: Sal & $-0,113+/-0,594$ \\
B: Aceite & $-0,590+/-0,594$ \\
AA & $0,962+/-1,029$ \\
AB & $1,104+/-0,728$ \\
BB & $1,154+/-1,029$ \\
\hline
\end{tabular}

La Tabla 12 muestra cada uno de los efectos estimados de la sal, aceite y de las interacciones entre ellas. Se observa que el Efecto Estimado de la sal $-0,113+/-0,594$, sobre el atributo "gusto de sal" es pequeño y su efecto no es significativo. El Diagrama de Pareto, Fig 4, muestra igualmente el bajo efecto de la sal.

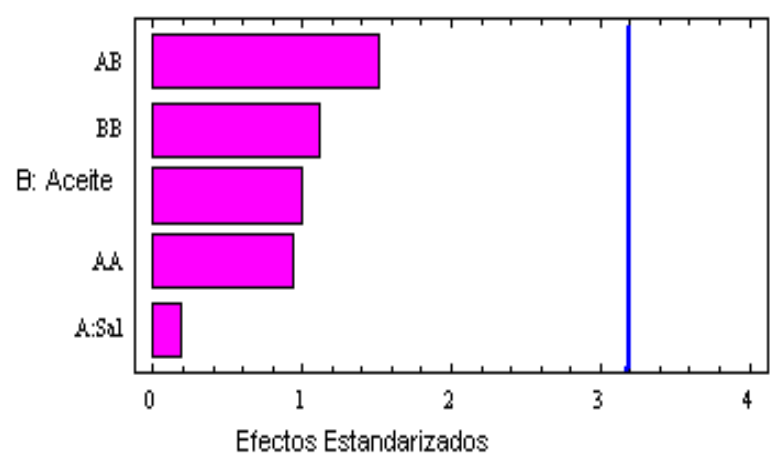

Figura 4 Diagrama de Pareto para el Atributo Gusto de Sal.

El ANOVA para el Atributo Gusto de Sal se muestra en la Tabla 13:
Tabla 13. Análisis de Varianza para el Atributo Gusto de Sal. Fuente Suma de Cuadrados GL Cuadrado Medio FC $\quad$ P-Val

\begin{tabular}{llllll}
\hline A:Sal & 0,019 & 1 & 0,019 & 0,04 & 0,861 \\
B:Aceite & 0,523 & 1 & 0,523 & 0,99 & 0,393 \\
AA & 0,462 & 1 & 0,462 & 0,87 & 0,419 \\
AB & 1,219 & 1 & 1,219 & 2,30 & 0,226 \\
BB & 0,665 & 1 & 0,665 & & 1,26
\end{tabular}

0,344

Total error

$1,590 \quad 3$

$3 \quad 0,530$

Total (corr.)

$4,478 \quad 8$

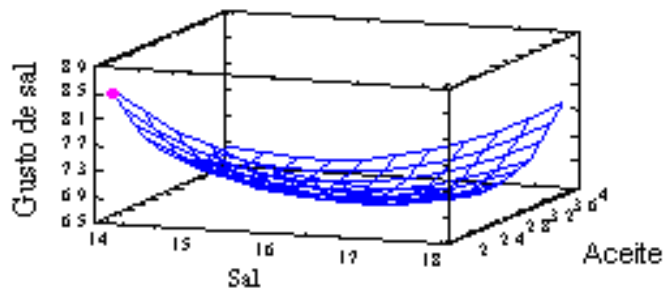

Figura 5. Superficie de Respuesta Estimada de Gusto de Sal.

Del análisis de varianza se observa que los P-Values son mayores a 0,05 , esto significa que no presentan diferencias significativas entre las interacciones a un intervalo de confianza del 95\%, se acepta la Hipótesis Nula, que no hay interacciones entre los niveles de sal y el de aceite añadidos. Asimismo en la Fig 5 se muestra a superficie de respuesta estimada de gusto de sal.

La Tabla 14 muestra los coeficientes de regresión que se han ajustado a los datos.

Tabla 14. Coeficiente de Regresión.

$\begin{array}{lll}\text { Constante } & = & 57,151 \\ \text { A:Sal } & = & -4,704 \\ \text { B:Aceite } & = & -8,175 \\ \text { AA } & = & 0,120 \\ \text { AB } & = & 0,276 \\ \text { BB } & = & 0,577\end{array}$

Cuya ecuación del modelo es la siguiente:

Gustod sal $=5,151-4,704[A]-8,175[B]+$ $0,120[A]^{2}+0,276[A][B]+0,577[B]^{2}$

Los valores de las variables son especificadas en sus unidades originales, Fig 6. 


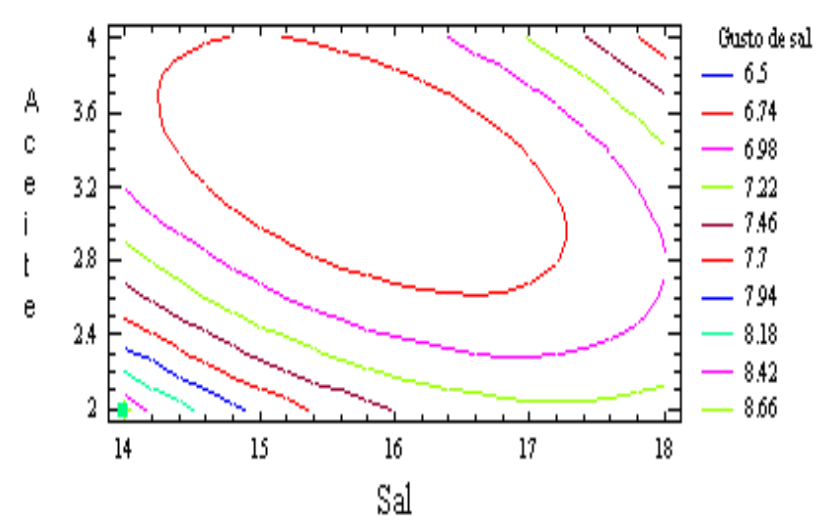

Figura 6. Contornos de la Superficie de Respuesta Estimada.

Optimizando las nueve formulaciones se muestra que el valor óptimo para el gusto de sal es 8,543 según la siguiente Tabla.

Tabla 15 Valor Óptimo para el Gusto de Sal.

\begin{tabular}{cccc}
\hline Factores & Mínimo & Máximo & Optimo \\
\hline Sal & 14 & 18 & 14 \\
Aceite & 2 & 4 & 2 \\
\hline
\end{tabular}

De acuerdo a la curva de nivel se muestra que el valor de gusto de sal y aceite es 14 y 2 respectivamente para estos valores la aceptabilidad es de 8,543.

\section{PRODUCTO FINAL ENRIQUECIDO}

Desarrollando el mismo procedimiento para las nueve formulaciones en lo referente a los atributos textura y apariencia, los resultados obtenidos confirman que el queso enriquecido correspondiente a la formulación F9 es el que posee los mejores atributos.

Haciendo uso de los valores óptimos obtenidos en el análisis estadístico se procedió a elaborar "el queso óptimo" enriquecido. El análisis sensorial de este queso otorgó los resultados que muestra la Fig 7.

De la Fig. 9 podemos deducir que la reducción de grasas saturadas se encuentra en la proporción 2,86: 1,0. Así mismo se puede notar el incremento considerable de ácidos grasos poliinsaturados de 1,0: 10,9 lo que demuestra un resultado óptimo.

Comparando estos resultados con los de la Fig 4, indica que los atributos de la formulación F9 son coincidentes. Su análisis químico del queso óptimo en la Tabla 16.

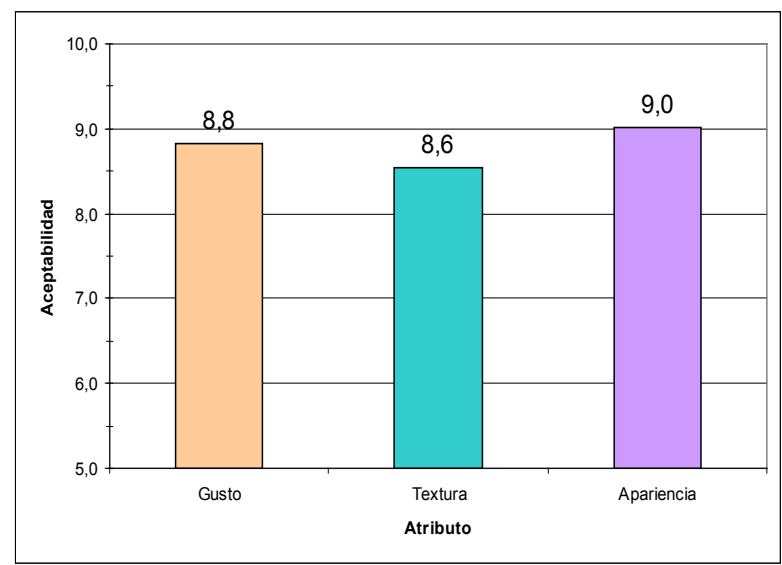

Figura 7. Aceptabilidad de la Formulación Óptima.

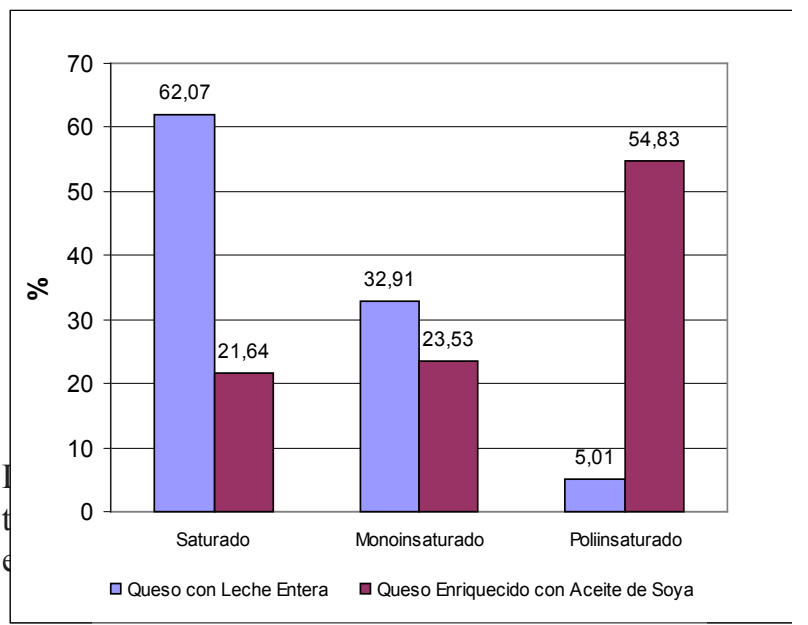

Figura 9 Composición de Ácidos Grasos en Queso Fresco enriquecido. 
Tabla 16. Composición de Ácidos Grasos de los Quesos con Leche Entera y Queso Enriquecido con Aceite de Soya.

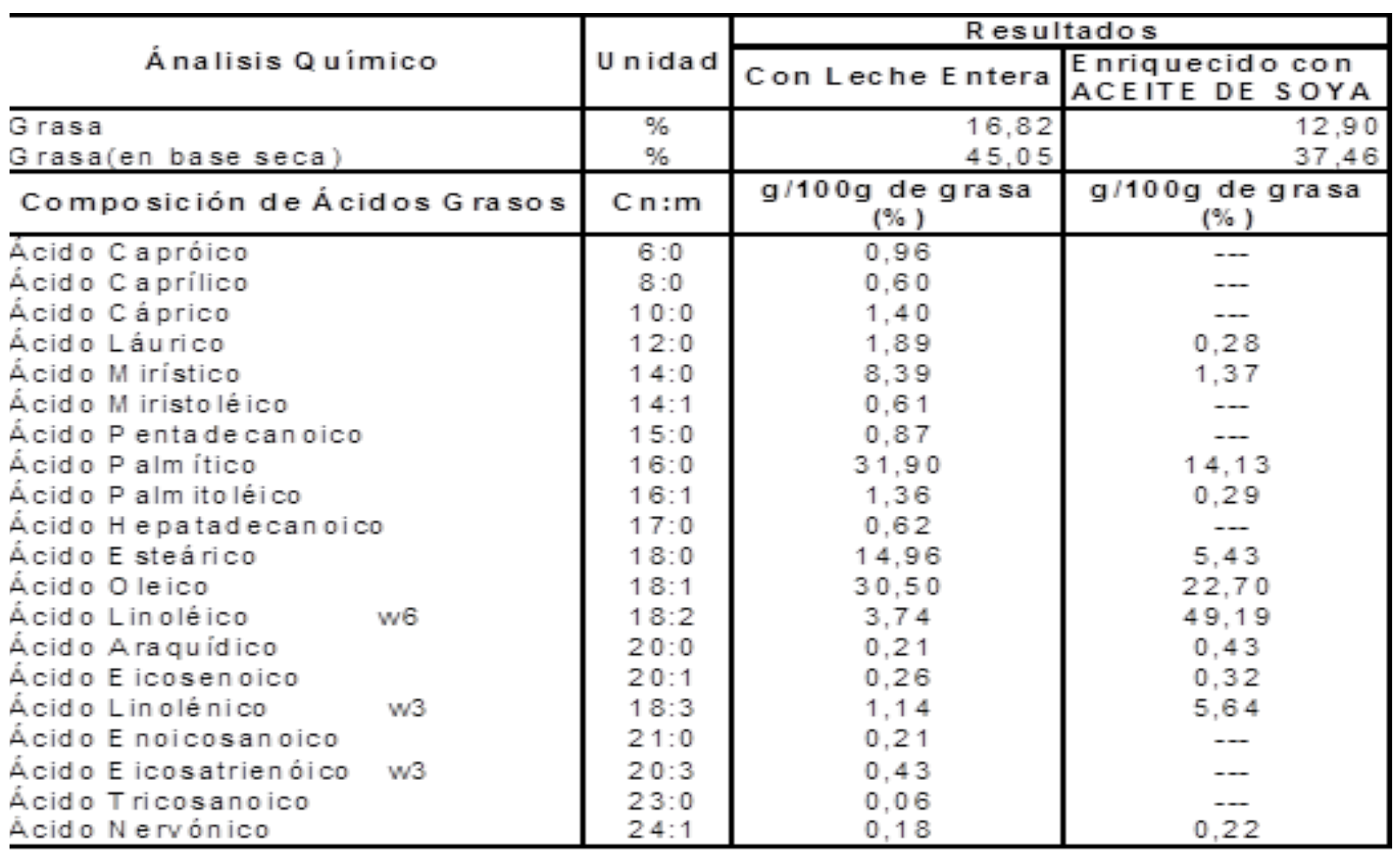

\begin{tabular}{l|c|c|}
\hline \multicolumn{1}{|c|}{ Análisis Químico } & $\mathrm{g} / 100 \mathrm{~g}$ de grasa \\
$(\%)$ & $g / 100 \mathrm{~g}$ de $\mathrm{grasa}$ \\
$(\%)$
\end{tabular}

\section{O b servaciones}

$n=$ nú m ero de carbonos

$\mathrm{m}=\mathrm{núm}$ ero de dobles enlaces

$w=0 \mathrm{mega}$

\section{Métodos}

Grasa: NTP 202.0721980 Queso

Composición de Ácidos G rasos: ISO 5509 - 1978

\section{Conclusiones}

$\mathrm{El}$ aceite de soya es una fuente importante de precursores de Omega-3 y Omega-6, 62,73\% ácidos grasos poliinsaturados, $22,13 \%$ monoinsaturados y $15,15 \%$ saturados.

Se puede incorporar ácidos grasos esenciales del aceite de soya al queso fresco de leche de vaca enriqueciendo su contenido en ácidos grasos poliinsaturados a 54,83\%, superior al que contiene el queso fresco elaborado con leche entera de $5,01 \%$.

La concentración de aceite de soya a ser adicionada para la elaboración del queso fresco enriquecido debe ser del $2 \%$.
La concentración óptima de sal es de $14 \mathrm{~g}$ de sal $/ \mathrm{L}$ de leche.

La etapa mas adecuada para la incorporación del aceite de soya debe ser entre las Etapas de Pasteurización y Mezcla Mecánica.

Se obtuvo, al análisis sensorial valores de: Sabor 8,870 , Textura 8,237 y Apariencia 9,013 de un máximo de 10,0 unidades lo que demuestra su aceptabilidad sensorial.

\section{Recomendaciones}

Sobre la base de los resultados obtenidos se propone lo siguiente: 
Se recomienda adicionar aceite de soya al queso fresco hecho con leche descremada de vaca para elevar su contenido en ácidos grasos poliinsaturados.

Efectuar estudios experimentales con otros aceites ricos en ácidos grasos esenciales tales como la Canola o el Sacha Inchi.

Se recomienda el estudio estadístico sobre el comportamiento del consumo en la dieta humana, especialmente sobre los efectos en los problemas cardiovasculares.

\section{Literatura citada}

Alais, Ch.1985. Ciencia de la Leche. España: Reverte, S.A.

Carey, F. 1999. Química Orgánica. Madrid: Mc Graw Hill.

Dubach, J. 1988. EL ABC Para La Quesería Rural de los Andes. Ecuador: ABC. Secretaría de Agricultura Ecuador.

Internacional Comission on Microbiological Specifications For Foods. 2000. 2da Ed. Vol 1 Part. E II, 1983 (Trad 1998) Reimp. 2000. Editorial Acribia.

ITINTEC.1982. № 202.087. Queso Fresco. Norma Técnica Peruana.

ITINTEC.1982. No 202.045. Queso. Extracción de Muestras. Norma Técnica Peruana.

ITINTEC. 1982. № 202.055. Queso. Determinación de acidez. Norma Técnica Peruana.

ITINTEC. 1982. No 202.072. Queso. Determinación de Materia Grasa. Método de Gerber Van Gulik. Norma Técnica Peruana.

ISO 5509-1978. 1978. Animal and vegetable fats and oils. Preparation of methyl esters of fatty acids.

ISO 5508-1990. 1990. Animal and fats and oils-Analysis by gas chromatography of methyl esters fatty acids.

Leighton, F. 2002 Dietas Mediterráneas 6(1): 1-20 Accesado En Boletín Ciencia Vino y Salud. http://www. bio.puc.cl7vinosalud/boletin/61mecani.htm.Junio2003

Meyer, M. 1999 Elaboración de Productos Lácteos. México: Trillas.

MINAG. 2007 Estadística Agraria Mensual. Perú: Oficina de Información Agraria.

MINSA. 2002. Situación de Salud en el Perú: Indicadores Básicos.

Montgomery. 2002 Diseño y Análisis de Experimentos. México: Limusa Wiley.

Padilla, A. 1999. Diferencias Regionales en los Patrones de Consumo en el Perú, Tercera Reunión del Grupo de Expertos en Estadísticas Sobre Pobreza (Grupo de Río), Lisboa.

Pérez, C. 1995. Análisis Estadístico con Statgraphics. España: RA-MA.

Pérez, R. 2003. El alimento que alarga la vida: Omega 3 \& Salud. Perú: Gráfica Cristal S.R.L.
Ureña, M., D'Arrigo, M. y Girón, O. 1999 Evaluación sensorial de los alimentos. Lima: Editorial Agraria.

Wade, Jr. 2004 Química Orgánica. Madrid: Pearson. 